\title{
Application of mesoporous silica nanoparticles as drug delivery carriers for chemotherapeutic agents
}

\section{Yasmine Alyassin ${ }^{1}$, Elshaimaa G. Sayed ${ }^{1,2}$, Prina Mehta ${ }^{1}$, Ketan Ruparelia ${ }^{1}$, Muhammad S. Arshad', Manoochehr Rasekh ${ }^{1,3}$, Jennifer Shepherd, Israfil Kucuk ${ }^{5}$, Philippe B. Wilson ${ }^{6}$, Neenu Singh ${ }^{1}$, Ming-Wei Chang ${ }^{7}$, Dimitrios G. Fatouros ${ }^{8}$ and Zeeshan Ahmad ${ }^{1}$}

\footnotetext{
${ }^{1}$ Leicester School of Pharmacy, De Montfort University, Leicester, LE1 9BH, UK

${ }^{2}$ Department of Pharmaceutics, Faculty of Pharmacy, Minia University, Minia, Egypt

${ }^{3}$ College of Engineering, Design and Physical Sciences, Brunel University London, Middlesex UB8 3PH, UK

${ }^{4}$ Department of Engineering, University of Leicester, Leicester, LE1 7RH, UK

${ }^{5}$ Institute of Nanotechnology, Gebze Technical University, 41400 Gebze, Turkey

${ }^{6}$ School of Animal, Rural and Environmental Sciences, Nottingham Trent University, Brackenhurst Campus, Southwell, NG25 0QF, UK

${ }^{7}$ Nanotechnology and Integrated Bioengineering Centre, University of Ulster, Jordanstown Campus, Newtownabbey, BT37 0QB, UK

${ }^{8}$ Department of Pharmaceutical Technology, Aristotle University of Thessaloniki, School of Pharmacy, 54124, Thessaloniki, Greece
}

Recently, remarkable efforts have focused on research towards enhancing and delivering efficacious and advanced therapeutic agents. Even though this involves significant challenges, innovative techniques and materials have been explored to overcome these. The advantageous properties of mesoporous silica nanoparticles (MSNs), such as unique morphologies and geometries, makes then favorable for use for various drug delivery targeting purposes, particularly in cancer therapy. As we discuss here, MSNs have been utilized over the past few decades to improve the efficiency of anticancer drugs by enhancing their solubility to render them suitable for application, reducing adverse effects, and improving their anticancer cytotoxic efficiency.

\section{Introduction}

Over recent years, various different studies have focused on the application of mesoporous silica-based platforms as effective nanocarriers in chemotherapy [1]. Mesoporous silica has favorable properties for use as a nanocarrier, such as large pore volume, large surface area, and adjustable pore morphological structures $[2,3]$.

The characteristics of inorganic silica (e.g., size, surface, and topology) can be altered to generate distinct interactions with different types of biological system. Thus, mesoporous silica, amorphous silica, microporous crystalline titanosilicates, and zeolites have been widely used in biomedical applications [4]. The desirable features of mesoporous inorganic materials, more specifically MSNs, are easily tailored to incorporate and interact effectively with an array of poorly

Corresponding author: Ahmad, Z. (zahmad@dmu.ac.uk) soluble drugs and biomolecules. thus, it is clear to see why there is a growing interest in this field [5,6]. Ordered MSNs are characterized by particle size $(50-200 \mathrm{~nm})$, pore sizes of $2-6 \mathrm{~nm}$, bulk pore volume of $0.6-1 \mathrm{~cm}^{3} / \mathrm{g}$ and a large surface area of $700-1000 \mathrm{~m}^{2} / \mathrm{g}$. Moreover, MSNs have the ability to bind to various kinds of functional groups of active pharmaceutical ingredients (APIs) to allow targeted delivery to the required site of action. These explicit characteristics render MSNs as promising nanocarriers that have revolutionized different drug delivery approaches [7], such as controlled [8], targeted [9,10], sustained [11], and responsive systems [12-14]. The characteristics of MSNs have been studied in depth with respect to their pharmacokinetic and immunological properties, which are major challenges to overcome to realize their potential in the clinic [15]. In this review, we discuss the different characteristics of MSNs and emphasize their involvement in recent advances in different drug delivery systems 
(DDSs), with a specific focus on their potential biomedical use in chemotherapy and cancer treatment.

\section{MSNs as a targeting delivery system for anticancer drugs}

Recent research has resulted in the applications of several targeting tools for use in drug discovery. Smart nanostructured vehicles have been utilized to improve the efficacy of anticancer drugs while reducing their nonselective adverse effects on nontarget tissues [16-19]. Different surface modification procedures have been used to achieve targeted localized delivery of anticancer agents to improve their efficiency in reducing tumor progression and their adverse effects [19]. MSNs have been used to successfully deliver different types of chemotherapeutic drug, including doxorubicin

TABLE 1

,

Applications of MSNs in cancer therapy

\begin{tabular}{|c|c|c|c|c|c|}
\hline Cancer type & Drug name & $\begin{array}{l}\text { Targeted cell } \\
\text { type }\end{array}$ & $\begin{array}{l}\text { Functionalized } \\
\text { mesoporous carrier }\end{array}$ & Pore size & Notes \\
\hline \multirow[t]{2}{*}{ Liver } & ATO & SC-7721 & LPMSNs & $7 \mathrm{~nm}$ & $\begin{array}{l}\text { In vitro assays showed huge cytotoxicity and } \\
\text { significant induction of apoptosis }\end{array}$ \\
\hline & Sorafenib & $\mathrm{HCC}$ & Gold nanoshell MSNs & Not stated & $\begin{array}{l}\text { Improved cancer suppression activity because of } \\
\text { higher accumulation of SO in hepatic tumor cells }\end{array}$ \\
\hline \multirow[t]{2}{*}{ Prostate } & DOX & LNCaP-AI & $\mathrm{CaCO}_{3}$ capped MSNs & Not stated & $\begin{array}{l}\text { Enhanced antitumor activity and premature } \\
\text { release stimulated under } \mathrm{pH} 7.4 \text { and fast drug } \\
\text { release under cancerous acidic environment }(\mathrm{pH} \\
6.5 \text { and 5.0). }\end{array}$ \\
\hline & DOX & LNCaP-AI & $\begin{array}{l}\text { PMSA surface- } \\
\text { modified MSN }\end{array}$ & $4 \mathrm{~nm}$ & $\begin{array}{l}\text { Improved cell internalization ( } \sim 25 \% \text { ) observed in } \\
\text { simulated physiological medium }\end{array}$ \\
\hline \multirow[t]{3}{*}{ Breast } & $\begin{array}{l}\text { Paclitaxel and } \\
\text { curcumin }\end{array}$ & $\begin{array}{l}\text { Canine breast } \\
\text { cancer cell line }\end{array}$ & $\begin{array}{l}\text { Lipid bilayer-coated } \\
\text { MSNs }\end{array}$ & $2.754 \mathrm{~nm}$ & $\begin{array}{l}\text { Bilipid layer improved loading of drugs by } \\
\text { enhancing their solubility, exhibited prolonged } \\
\text { release and high cytotoxic activity against breast } \\
\text { cancer }\end{array}$ \\
\hline & ATZ & MCF-7 & $\begin{array}{l}\text { Chitosan folate- } \\
\text { capped MSN-41 type } \\
\text { MSNs }\end{array}$ & $3.36 \mathrm{~nm}$ & $\begin{array}{l}\text { pH-responsive targeting; in vitro drug release } \\
\text { profile exhibited controlled pH-responsive and } \\
\text { enhanced drug release rate; cytotoxic against } \\
\text { breast cancerous cells }\end{array}$ \\
\hline & DOX & MCF-7 cell line & $\begin{array}{l}\text { MSN-coated gold } \\
\text { nanorods }\end{array}$ & Not stated & $\begin{array}{l}\text { Synergistic effect; chemotherapeutic effect of } \\
\text { DOX loaded in mesoporous shell; photothermal } \\
\text { effect of gold core; resulted in significant damage } \\
\text { to cancer cells }\end{array}$ \\
\hline \multirow[t]{2}{*}{ Lung } & Cisplatin and DOX & $\mathrm{BCL} 2$ and MRP1 & PEG-modified MSNs & Not stated & $\begin{array}{l}\text { Enhanced cytotoxicity and induction of selective } \\
\text { apoptosis resulting from co-delivery of cisplatin } \\
\text { and DOX simultaneously with BCL2 and MRP1 } \\
\text { siRNAs. In vivo assays showed MSNs were inhaled } \\
\text { through lungs, avoiding their uptake by systemic } \\
\text { circulation }\end{array}$ \\
\hline & Bortezomib & $\begin{array}{l}\text { A549 cells (p53 } \\
\text { wild-type) and }\end{array}$ & HMSNs & $4.1 \mathrm{~nm}$ & $\begin{array}{l}\text { Synergetic effect of bortezomib and HMSNs } \\
\text { demonstrated improved tumor apoptosis }\end{array}$ \\
\hline
\end{tabular}

Refs

H1299 cells ( $p 53$ mutant)

Colon

DOX

Human mesenchymal stem cells

5-FU

HT-29

PAA-functionalized

$7.8 \mathrm{~nm}$

In vitro studies confirmed high loading drug

SBA-15-type MSNs

Guar gum-capped

adenocarcinoma

cell line

Brain

DOX

U-87 MG-luc2 cells

Tf-modified MSNs

U87 glioma cells

Functionalized MSNs with arginylglycylaspartic acid peptide

Bone

MC3T3-E1

preosteoblastic PAA-capped MSNs

$2.4 \mathrm{~nm}$

Not stated capacity ( $785.7 \mathrm{mg} / \mathrm{g})$, excellent compatibility and good $\mathrm{pH}$-triggered response

$2.9 \mathrm{~nm} \quad$ Guar gum capping used as effective in vivo enzyme-responsive carrier

Tf-magnetic field resulted in high drug delivery capacity with enhanced cytotoxicity and release profile against cancerous cells compared with normal cells

Tailoring particle size and functionalization of MSNs achieved improved efficacy and targeting against glioma cell lines

PAA acted as gatekeeper, reducing premature release of drug and resulting in $\mathrm{pH}$-responsive release in tumor target site; ligand-selective binding to cancer cells overexpressing glycans (e. g., sialic acids)

WMSNs
A new approach to using MSNs in detection of early-stage ovarian cancer using nanostructured formulations 
(DOX) [20] and paclitaxel [21], which resulted in inhibitory effects on tumors. Table 1 highlights the range of MSN applications in cancer therapy.

Three distinct approaches have been developed when utilizing MSNs: passive targeting, active (cell-selective) targeting, and controlled stimulus-responsive release targeting [22]. Several studies have shown the defective structure of blood vessels in tumors tissues, which allows different vascular permeability in cancer cells. Therefore, most solid tumors are characterized by a degree of vascular permeability, which provides a suitable supply of oxygen and nutrients that enable the fast growth of cancerous tissues. This is known as the enhanced permeability and retention (EPR) effect [23]. As a result of this effect, any macromolecules ( $>40 \mathrm{kDa}$ ) can selectively escape from cancerous blood vessels and accumulate in cancerous tissues [24]. By contrast, the EPR effect does not occur in normal tissues. Thus, in solid tumor tissues, the EPR effect can be a target for chemotherapeutic agents and is increasingly emerging in drug delivery science as a promising clinical treatment [23].

\section{Passive targeting}

To accomplish effective targeting delivery of NPs, it is crucial to extend their circulation time through the mononuclear phagocytic system (MPS) and decrease their rate of renal clearance. Therefore, it is essential for nanoparticles to be $10 \mathrm{~nm}$ diameter and 100 $200 \mathrm{~nm}$ size [25] to penetrate the tumor tissues via passive diffusion and to avoid the MPS [26,27]. Meng et al. reported effective passive targeting using polyethyleneimine-polyethylene glycol (PEG)-functionalized MSNs with a particle size of $50 \mathrm{~nm}$ [28]. The results indicated an improvement in DOX delivery through passive diffusion to cancerous cells. This system not only achieved the EPR effect, but also improved DOX cellular uptake by tumor cells by using a nanostructured particulate system. Therefore, it preferably induced cellular apoptosis and tumor size reduction alongside avoiding severe DOX cytotoxic effects [28]. Another study demonstrated that mesoporous silica-coated gold nanorods showed an efficient passive targeting effect [29]. This unique nanodevice exerted two synergistic therapeutic effects; the chemotherapeutic effect of DOX loaded in the mesoporous shell and the photothermal effect of the gold core. This nanosystem achieved exceptional results compared with pure DOX, including targeting tumor tissues, inducing damage to Ehrlich ascites carcinoma in vivo, causing significant cytotoxicity to a breast cancer cell line (MCF-7), and decreasing cytotoxic effects on normal tissues [29].

\section{Active targeting}

Active targeting can be used to improve the therapeutic efficiency of a drug by overcoming the challenges associated with passive targeting systems, such as the lack of the ability to control entrapped API release and reduced tumor specificity. In active targeting, specific ligands are conjugated to MSNs via surface modification. Ligand-modified MSNs tend to recognize receptors that are selectively expressed on the membrane of cancerous cells via ligand-receptor interactions, therefore improving the antitumor selectivity $[26,27]$. The ligands could be peptides [30], aptamers [31], antibodies [32], proteins [33], saccharides [34], and folic acid [35]. Active targeting offers a pathway for selective localized delivery of anticancer drugs to tumor cells, causing efficient cellular endocytosis [6]. Bioactive molecules, such as folic acid, have been used extensively to deliver several anticancer therapeutics to different kinds of tumor (i.e., those that overexpress folate receptors on their surfaces) including lung, ovarian, breast, kidney, endometrial, colon, and brain cancers [36]. A recent study developed a smart device compromising functionalized hyaluronic acid (HA) MCM-41-type MSNs to achieve active targeting utilizing two different molecular weights of HA (6.4 and $200 \mathrm{kDa})$. The study demonstrated that optimizing different parameters, including the method of loading and molecular weight of HA, resulted in the enhanced stability and dispersity of MSNs in biological fluids. It indicated that high-molecular-weight HA-functionalized MSNs demonstrated high biocompatibility, low cytotoxicity, and favorable targeted binding to HA receptors (CD44) that are overexpressed in many cancerous cells. These results suggest that HAfunctionalized MSNs are a promising platform for cancer-targeting therapy [10].

\section{Responsive targeting systems}

Stimuli-responsive systems are an advanced strategy that provides on-demand release of therapeutic molecules in response to external or internal stimuli [22]. The on-demand release of APIs inside specific intracellular compartments can be only achieved as a response to explicit endogenous or exogenous changes in the microenvironment of the cells [37]. These changes can be triggered by external stimuli (e.g., electricity, temperature, ultrasound, light, and magnetism) and internal stimuli (e.g., enzymes, reactive oxygen species, redox potential, $\mathrm{pH}$, and ionic strength). Nevertheless, on-demand release (which is triggered by intracellular stimuli) is a more suitable pathway for clinical applications [38]. Here, MSNs are utilized to protect cargo molecules from premature release and only allow their release upon exposure to stimuli that are selectively present in the tumor tissues [39], such as acidic $\mathrm{pH}$ [13] and enzymes [20,40]. Many studies have used this responsive release strategy to improve the treatment outcomes of chemotherapy, including its safety and efficacy [6]. Three essential routes can be utilized to achieve on-demand release: modifying the surface of MSNs via a responsive polymer coating [41], attaching certain ligands (gatekeeper) to their mesopores [42], or anchoring the anticancer agent to MSNs via responsive cleavable linkers [43].

The flexibility of MSN synthesis techniques has enabled their layer-by-layer construction to result in mate4rial of an accurate thickness, which results in advantages including permeability properties and elasticity $[44,45]$. The delivery of their cargo depends on interactions between molecular charges. When the $\mathrm{pH}$ is neutral, charges are electrostatically stable and the layers stay locked, which maintains the cargo within the layers and avoids its premature release. Once the $\mathrm{pH}$ shifts, the molecular charges react, destroying the multilayered assemble to release the cargo for targeted delivery [46].

Protein regulation and metabolism result in acidic cancerous tissues, with a pH range of 4-6.5 [47]. The difference in $\mathrm{pH}$ between normal and cancerous cells can be useful in targeting drug delivery [39]. The flexible MSN structure allows different molecules to attach to their surface for specific targeting [48]. Functionalized MSNs can be modified chemically via degradation or charge conversion as a response to $\mathrm{pH}$ changes. These drug carriers tend 
to be stable in a neutral $\mathrm{pH}$ environment ( $\mathrm{pH}$ 7.4) and degrade upon exposure to an acidic $\mathrm{pH}$ environment (e.g., tumor tissues) [47]. Thus, MSNs have been exploited using this approach to increase cellular uptake and to reduce the adverse effects of chemotherapy. For example, an effective nanocarrier was developed using conjugated MSNs to target bone cancer by exploiting their pH-responsive properties [14]. A novel device of DOX-loaded MSNs was formed that was coated with a polymeric shell (polyacrylic acid; PAA) via acid degradable linkage. PAA acted as a gatekeeper to reduce the premature release of DOX but to allow its release in response to changes in $\mathrm{pH}$ in the tumor site. A synergist active targeting effect was provided by grafting lectin concanavalin A into MSNs. This targeting ligand selectivity binds to glycan (e.g., sialic acids)-overexpressing cancer cells. In vitro studies showed that this novel DOX-loaded MSNs increased the anticancer efficacy of DOX up to eightfold compared with free drug [14].

MSNs are not only used for cancer therapy, but are also being explored as a promising diagnostic approach to assist clinicians in detecting tumors at earlier stages of disease. A recent study demonstrated wormhole-shaped mesoporous silica nanoparticles (WMSNs) for the diagnosis and detection of ovarian cancer owing to their ability to target tumor tissues. With a diameter of $27 \mathrm{~nm}$, these MSNs were produced by sol-gel process and were loaded with imaging probes for diagnosis purpose. WMSNs were functionalized with chitosan and a V7 pHLIP peptide to achieve the $\mathrm{pH}$ responsive release of the imaging probes only inside the acidic environment of ovarian tumors, preventing their off-target premature release. Intravenous injection of this complex in mice allowed the early detection of primary-stage ovarian tumor. Thus, the study highlighted how nanostructured formulations can be explored to discover tumors using constantly evolving imaging technology [13].

\section{MSN applications in chemotherapy}

Cancer is a significant cause of morbidity in humans. Although chemotherapy is considered to be the most potent anticancer treatment, it does not destroy cancer tissues specifically, but instead also affects noncancerous tissues [49]. To overcome this issue, researchers have developed cargo delivery systems to increase the drug concentration in tumor tissues and to improve the delivery of the drug molecules to the target area $[6,15]$.

The most crucial aspect of chemotherapy is the use of different chemical entities to induce cellular apoptosis. However, the systemic administration of cytotoxic therapeutic agents results in cellular death in both cancerous and healthy tissue [1]. Given the deficiency of selectivity of chemotherapeutic agents, severe adverse effects [e.g., hair loss, myelosuppression, cardiotoxicity, immunosuppression, mucositis (gastrointestinal tract inflammation) and neurotoxicity] can be induced [50], resulting in decreased patient compliance. For example, the use of the anticancer drugs paclitaxel and docetaxel (derived from natural sources and used for the treatment of solid tumors [51]) has been restricted because of their cardiotoxicity effects and poor aqueous solubility $[22,52]$. As a result, there have been various attempts to enhance the therapeutic profiles of these cytotoxic compounds and increase their applicability. In addition to toxicity, conventional chemotherapy also suffers from the poor solubility of hy- drophobic drugs, fast systemic elimination, and multidrug resistance (MDR) [49]. MDR is defined by the ability of drugresistant tumors to express resistance simultaneously against several functional and structural unrelated anticancer compounds [53]. Therefore, research has focused extensively on developing nanostructured chemotherapeutics carriers that are capable of achieving anticancer on-site delivery to address MDR $[1,54]$, resulting in various biomedical applications [22]. Among different inorganic nanomaterials, MSNs have attracted significant interest as effective vehicles in anticancer drug delivery because of their flexible in vivo and in vitro properties [42]. The discovery of silicabased nanoparticles was an important milestone in cancer therapy owing to their favorable characteristics, such as biocompatibility, chemical and thermal stability, tunable pore size, high pore volume, and ease of surface area alteration [36]. In addition, most antitumor drugs demonstrate poor water solubility, poor permeability across biological membranes, and inadequate bioavailability that restricts their administration by intravenous or oral routes. Thus, MSNs have been used to improve their solubility and enhance their permeability [55], resulting in a better accumulation of anticancer drugs at the tumor site, improving their efficiency $[12,16,46,55]$.

Moreover, the structures of MSNs can be modified via diverse stimuli-responsive gatekeepers to enhance drug release. In addition, ordered MSNs are able to carry a high payload, thus ensuring controlled delivery for chemotherapeutics to cancerous tissues. Their nanosize enables the EPR effect and their surface-grafted ligands allow active targeting of specific molecular structures on the surface of the cancer cells [22].

\section{MSNs in liver cancer}

Despite significant advances in treatments, mortality resulting from liver cancer remains high worldwide [56]. There has been much interest in the use of nanocarriers as a DDS to improve the treatment of hepatic cancer. For example, arsenic trioxide (ATO), an antileukemia drug, has been approved by the FDA as a chemotherapeutic agent for solid tumors. Chi et al. encapsulated ATO prodrugs into the pores of large-pore MSNs (LPMSNs) to treat hepatocellular carcinoma (HCC). The authors reported real-time monitoring using magnetic resonance imaging by inserting magnetic iron oxide NPs into the MSN pores [57]. Stimuli-responsive targeting was achieved by modification of the silica surface using folic acid ligand (M-LPMSN-NiAsOx-FA). In vitro assays demonstrated improved cytotoxicity efficacy compared with free ATO because of a significant induction of apoptosis in SC-7721 cells. Furthermore, in vivo showed a controlled release delivery system through the imaging ability of M-LPMSN-NiAsOx-FA. Thus, this work highlights the versatility of MSN-based platforms [57].

Another report indicated a potential candidate for HCC treatment and tumor apoptosis via a combined chemo/photothermal line therapy based on a MSN platform. A novel design of sorafenib (SO), an antitumor agent and first-line HCC therapy, was developed in this study. SO-Au-MSNs were obtained with a gold (Au) nanoshell for photothermal conversion. The authors reported improved cancer suppression activity as a result of the higher accumulation of SO in hepatic tumor cells. In response to near-infrared radiation, the synergistic chemo/photothermal effect resulted in a higher drug absorption rate and enhanced cytotoxicity of SO [58]. 
Thus, multifunctional designated MSNs have resulted in increased tumor death with respect to HCC, reducing the adverse effects of the API while achieving its controlled release.

\section{MSNs in breast cancer}

MSNs have been explored to enhance the solubility of therapeutic agents for use against breast cancer [52]. Given the obstacles of administrating antitumor agents (including solubility and stability), new tools have emerged to overcome these difficulties. For example, some researchers used lipid bilayer-coated MSNs to codeliver two anticancer drugs (paclitaxel and curcumin) intravenously to breast tumors with the aim of enhancing their bioavailability and reducing adverse effects. Both drugs exhibit low aqueous solubility and permeability, which results in their poor bioavailability and adverse effects, limiting their therapeutic use. Loading these agents into lipid bilayer-coated MSNs improved their solubility, achieving a sustained release and enhancing their cytotoxicity against breast cancer. The excellent dispersibility and high surface area of the lipid bilayer-coated MSNs also allowed the intravenous injection of the hydrophobic anticancer drugs [21].

In addition, $\mathrm{pH}$-responsive MSNs have been developed for the delivery of anastrozole (ATZ) to overcome traditional problems associating with chemotherapeutic drugs. The synthesis of MSNs was accomplished to attain the carboxylic functional group $\mathrm{CH}$. For this purpose, ATZ was loaded into the pores of the MSNs, which were capped with a chitosan-folate conjugate (MSN-ATZCH-FA) (Fig. 1a). The in vitro drug release study demonstrated a controlled $\mathrm{pH}$-responsive and improved drug release profile, while being selectively more cytotoxic to breast cancer cell lines. The study also highlighted the usefulness of MSN engineered-based systems as effective in vivo $\mathrm{pH}$-responsive carriers that can restrict cancer metastasis [59].

Surface modification of MSNs resulted in significantly improved cargo bioavailability and cytotoxicity of the encapsulated drugs against breast cancer with a sustained release profile. Thus, MSNbased DDSs are considered to be promising carriers for breast tumor therapies.

\section{MSNs in prostate cancer}

Prostate cancer is one of the most frequent tumors affecting men. The treatment of prostate cancer has improved over the past few years, leading to an increase in survival rates. However, it remains a serious cause of death in several regions [60]. Owing to the beneficial properties of MSNs as DDSs, hydrophobic cargo mole-
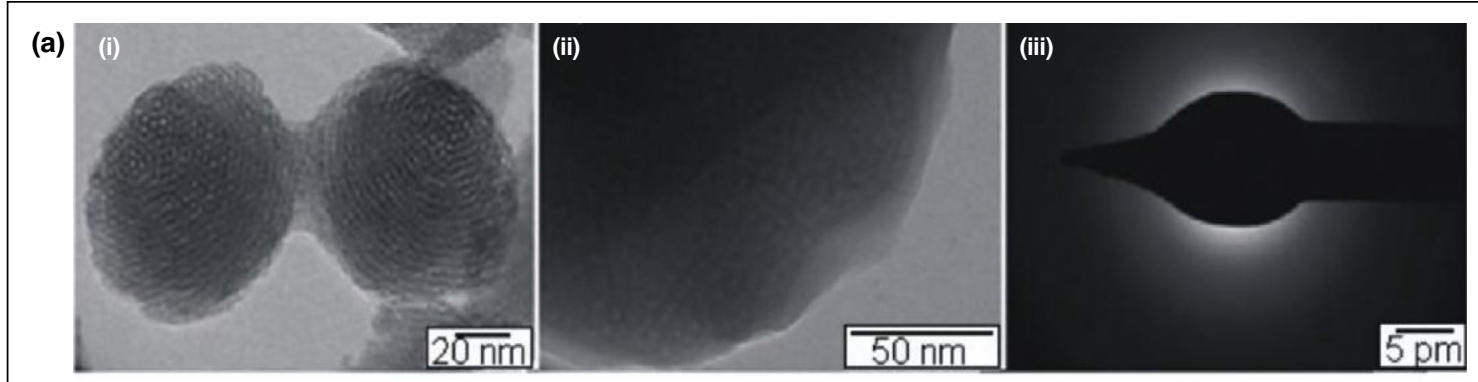

(b)
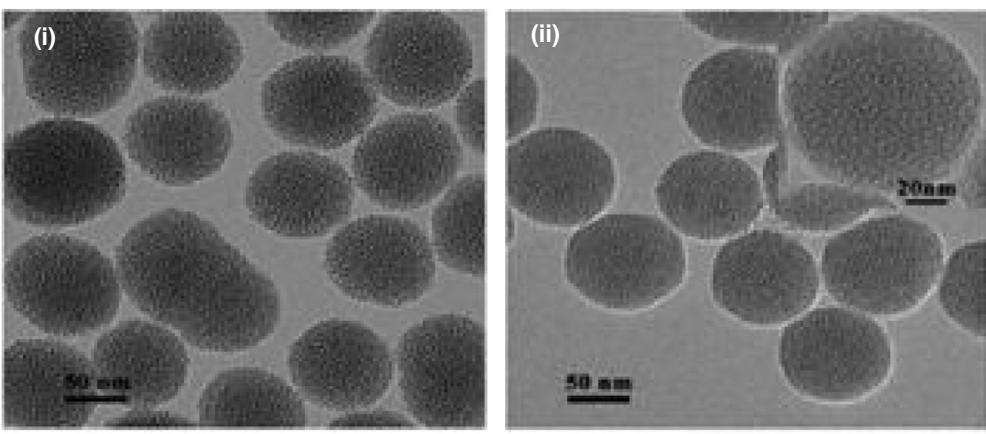

(c)
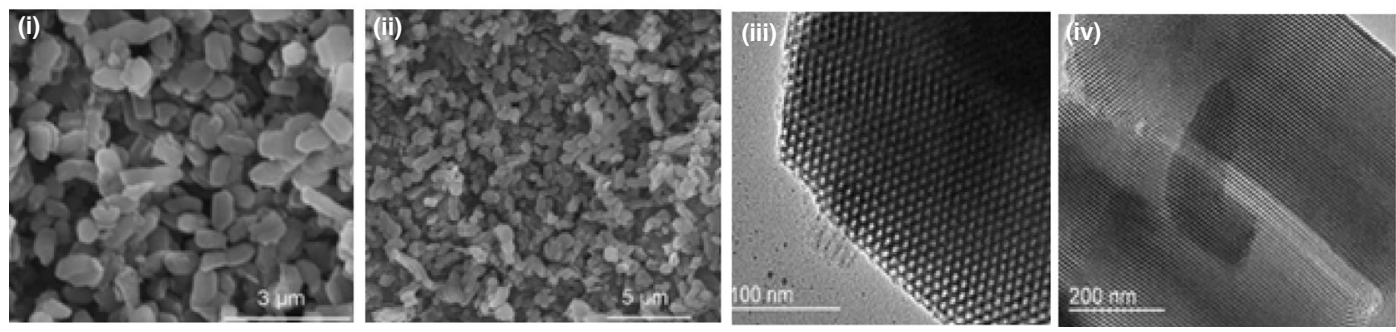

$\overline{\text { Drug Discovery Today }}$

\section{FIGURE 1}

Examples of mesoporous silica nanoparticles (MSNs) in chemotherapy. (a) Transmission electron microscopy (TEM) images of MSNs (i), MSN-anastrozole (ATZ)chitosan (CH)-folate (FA) (ii); selected area (electron) diffraction (SAED) image of MSNs (iii) [56]. (b) TEM of MSN (i) and doxorubicin (DOX)/MSN@CaCO 3 (ii) [59]. (c) Scanning electron microscopy (SEM) images (i,ii) and TEM images (iii,iv) of SBA-15 [63]. 
cules can be attached to their pores for targeted delivery. To this end, different studies have applied conjugated antibody/NP systems to treat prostate cancer. Despite the evolution of DDSs, there have been no published reports of the use of MSNs as targeted therapy against prostate tumors. However, research has been reported on the administration of particular antibodies by MSNs [61].

For example, Liu et al. successfully synthesized a biocompatible encapsulated core/shell designed NP to promote tumor apoptosis in prostate cancer cells (LNCaP-AI). In this study, DOX/ MSNs@CaCO $\mathrm{CO}_{3}$ was covered with an outer layer of cancerous cell membrane (CM) to enable the MSNs to enter and accumulate at high levels within, the tumor site. The surface modification of MSN@CACO ${ }_{3}$ was introduced as a detachable pH-sensitive stimulus for controlling DOX release without affecting the morphology of the NP (Fig. 1b). Premature release was stimulated under $\mathrm{pH} 7.4$ and fast DOX release under cancerous acidic environment $(\mathrm{pH} 6.5$ and 5.0), showing better anticancer activity compared with free DOX [62].

Another innovative drug delivery system was developed by Rivero-Buceta et al., who reported a stable conjugated ligand of DOX with an antiprostate-specific membrane antigen (PSMA) molecule (anti-FOLH1 monoclonal antibody, clone C803 N) in MSNs against LNCaP-AI cells. These MSNs demonstrated better cell internalization ( $25 \%)$ in a simulated physiological medium. Moreover, the cytotoxicity of DOX increased twofold compared with untargeted NPs and free DOX. By contrast, nonbearing-PSMA and PC3 cell lines showed a lack of targeting efficiency. Therefore, this study highlights the potential use of MSNs against non-metastatic prostate cancer [63].

\section{MSNs in lung cancer}

Annually, almost 220000 people are found to have lung cancer in the USA. There are two different kinds of lung cancer; non-small cell lung cancer (NSCLC) and small cell lung cancer (SCLC). Significant progress has been made in this field using MSNs, which can be administrated via the inhalation route to deliver drugs to lungs to achieve local targeted action.

For example, the delivery of cisplatin and DOX simultaneously with BCL2 and MRP1 small interfering (si)RNAs to lungs has been accomplished using MSNs. This co-delivery system is administered via inhalation to target lung carcinoma. Additionally, a targeting peptide (LHRH) was attached to the MSN surface using PEG as a linker. This unique combination inhibited both pump and nonpump-resistance mechanisms, enhancing the cytotoxicity of the loaded anticancer drugs and inducing selective apoptosis of NSCLC. In vivo administration of MSNs through inhalation achieved a local preferential targeting action in murine lungs and avoided their escape to the systemic circulation. This was evidenced by accumulation of $73 \%$ of MSNs in the lungs and their absence or minor presence in other tissues (spleen, heart, kidney, and liver). By contrast, intravenous administration of MSNs caused serious accumulation of the NPs in liver (73\%), with only 5\% being found in lungs [64].

Another study established a nanocarrier delivery system including the encapsulation of bortezomib (a proteasome inhibitor) and p35 into hollow MSNs (HMSNs) for the treatment of NSCLC. The study enhanced the efficacy of the encapsulated drug and prompted p35 signal pathways. Given the synergetic effect of bortezomib and HMSNs, tumor apoptosis was clearly achieved. p35 exhibited slow release under normal physiological conditions and very fast under acidic conditions (lysosomes and endosomes) [65].

Thus, advances in DDSs based on MSN nanocarriers have resulted in local targeted delivery via inhalation, improved accumulation of anticancer drugs in the target area, and successful gene delivery to tumor sites, achieving tumor suppression.

\section{MSNs in colon cancer}

The application of capped MSNs has achieved successful treatment of colon cancer using the oral route because of their ability to enhance the solubility of hydrophobic anticancer drugs targeting colon carcinomas, preventing premature drug release [66]. For example, a pH-triggered nanodevice was developed based on PAAfunctionalized SBA-15 type MSNs (Fig. 1c). PAA acted as a gatekeeper to retain DOX molecules within SBA-15 mesopores during their transportation to the target site. Under gastric conditions ( $\mathrm{pH} 2.0$ ), DOX was protected from premature leakage whereas, under colonic conditions ( $\mathrm{pH}$ 7.6), DOX demonstrated immediate release. In vitro studies showed a high loading drug capacity $(\sim 785.7 \mathrm{mg} / \mathrm{g})$, excellent compatibility, and a good $\mathrm{pH}$-triggered response. Furthermore, this study indicated the improved solubility of DOX molecules in the colonic environment [66].

Kumar et al. investigated a colon cancer treatment using MCM41-type MSNs to increase the effectiveness of 5-flurouracil (5-FU) using an enzyme-responsive system. They functionalized MSNs with a natural polymer (guar gum) to retain the drug inside mesoporous channels under physiological conditions. 5-FU release was activated through the degradation of the guar gum cap in response to colonic enzymatic activity. The release of the anticancer agent was around zero when the enzymes were absent in different gastrointestinal tract conditions. thus, the study highlights another significant form of MSN engineered-based systems using guar gum capping as an effective in vivo enzyme-responsive carrier [67].

\section{MSNs in brain cancer}

Although there has been great progress in the detection of different cancers, many challenges remain associated with brain cancers. A major form of brain cancer is a high-grade malignant glioma, glioblastoma multiforme (GBM), which is defined by the successive growth and immediate damage of brain parenchyma. Given its strong resistance to chemotherapy, fast cell damage, intense frequency of relapse, and poor survival rates, GBM is classified as a life-threatening tumor [68]. Step-by-step exploration of mesoporous silica materials has enabled the development of an innovative drug delivery platform for use against GBM [36].

A study reported improved anticancer drug delivery to GBM by using protein-grafted MSNs. Transferrin (Tf), a biological blood glycoprotein, was added to the external surface of the MSNs with conjugated magnetic silica poly(d,l-lactic-co-glycolic acid) NPs (MNP-MSN-PLGA-Tf NPs). In these NPs, Tf is considered as both the gatekeeper and the targeting agent to achieve the better therapeutic efficiency of DOX The abundant presence of the Tfmagnetic field resulted in DOX being delivered with higher cyto- 
toxicity and enhanced release rate compared with free DOX. Thus, Tf-modified MSNs are a potential carrier system that can suppress tumor growth, resulting in a selective cytotoxic effect against GBM and reducing systemic adverse effects [69].

Mo et al. tailored the size of MSNs to successfully cross the blood-brain barrier to target GBM. The authors fabricated different-sized MSNs (20,40, and $80 \mathrm{~nm}$ ) with capped arginylglycylaspartic acid peptide cRGD-conjugated DOX. This allowed the MSNs to selectively attach to U87 cells with a large $\alpha \nu \beta 3$ integrin and improved cellular uptake using a particle size of $40 \mathrm{~nm}$. Thus, amending the particle size and functionalization of MSNs could be an effective approach to target GBM via a sequentially enhanced cancer-targeting effect [70].

\section{Concluding remarks and perspectives}

Despite significant research on MSNs and their potential as a cancer therapeutic, there remains a lack of MSNs in clinic practice owing to the their inability to be successfully delivered to and accepted by living organisms hence hindering the process to clinical trials [71]. Nevertheless, they have shown great progress in their use as imaging systems and theranostics, specifically in aiding the diagnosis of ovarian cancers [13].

Various technologies are involved in the engineering of these carriers. Conventional methods, such as solvent impregnation, are common fabrication methods that have successfully yielded MSNs $[5,32]$. More recent emerging engineering technologies, such as

electrohydrodynamic atomization (EHDA), have started to be exploited to develop MSNs in a simple one-step, cost-,effective method [72,73]. By utilizing ever-evolving technologies, these beneficial carriers can be continuously fabricated in a cost-effective, easily adaptable, and modified way that can be altered to fit specific requirements or criteria, rendering them attractive for personalized drug delivery and therapy.

This review focused on recent applications of MSNs from a chemotherapy prospective. In addition, it highlighted the relevant characteristics of MSNs, such as adjustable pore size, high surface area, bioactivity, and release properties, and the different types of technique used, to deliver safe and compatible anticancer APIs. Despite the challenges associated with delivering a drug, significant progress has been made in the biomedical field, particularly with MSNs. Moreover, numerous methods can be used to aid MSN-surface modifications to enhance the responsive release of drugs and control drug delivery. MSNs can be also used in optical imaging to in diagnose tumors at early stages of disease. Thus, effective MSN-based platforms could in the future overcome the disadvantages associated with current stand-of-care chemotherapy.

\section{Conflict of Interest}

The authors declare that they have no known competing financial interests or personal relationships that could have appeared to influence the work reported in this paper.

\section{Acknowledgments}

The authors would like to thank the Council for At-Risk Academics (CARA) and the Egyptian Culture Centre and the Educational Bureau in London for supporting this work.

\section{References}

1 Castillo, R.R. et al. (2017) Advances in mesoporous silica-based nanocarriers for codelivery and combination therapy against cancer. Expert Opin. Drug Deliv. 14, 229 243

2 Sayed, E. et al. (2017) Porous inorganic drug delivery systems-a review. AAPS PharmSciTech 18, 1507-1525

3 Vallet-Regí, M. et al. (2017) Mesoporous silica nanoparticles for drug delivery: current insights. Molecules 23, 47

4 Vilaça, N. et al. (2017) Comparison of different silica microporous structures as drug delivery systems for in vitro models of solid tumors. RSC Adv. 7, 13104-13111

5 Li, Z. et al. (2019) Mesoporous silica nanoparticles: synthesis, classification, drug loading, pharmacokinetics, biocompatibility, and application in drug delivery. Expert Opin. Drug Deliv. 16, 219-237

6 Navya, P.N. et al. (2019) Current trends and challenges in cancer management and therapy using designer nanomaterials. Nano Converg. 6, 23

7 Castillo, R.R. and Vallet-Regí, M. (2019) Functional mesoporous silica nanocomposites: biomedical applications and biosafety. Int. J. Mol. Sci. 20, 929

8 Gonzalez, G. et al. (2018) Comparative study of two silica mesoporous materials (SBA-16 and SBA-15) modified with a hydroxyapatite layer for clindamycin controlled delivery. Microporous Mesoporous Mater. 256, 251-265

9 Montalvo-Quiros, S. et al. (2019) Cancer cell targeting and therapeutic delivery of silver nanoparticles by mesoporous silica nanocarriers: Insights into the action mechanisms using quantitative proteomics. Nanoscale 11, 4531-4545

10 Ricci, V. et al. (2018) Hyaluronated mesoporous silica nanoparticles for active targeting: Influence of conjugation method and hyaluronic acid molecular weight on the nanovector properties. J. Colloid Interface Sci. 516, 484-497

11 Rehman, F. et al. (2017) Amine bridges grafted mesoporous silica, as a prolonged/ controlled drug release system for the enhanced therapeutic effect of short life drugs. Mater. Sci. Eng. C 72, 34-41

12 Chen, C. et al. (2019) A self-targeting and controllable drug delivery system constituting mesoporous silica nanoparticles fabricated with a multi-stimuli responsive chitosan-based thin film layer. Int. J. Biol. Macromol. 122, 1090-1099

13 Samykutty, A. et al. (2018) Acidic tumor microenvironment targeted wormholeshaped mesoporous silica nanoparticles to detect ovarian cancer by multispectral optoacoustic tomography. Cancer Res. 78 (Suppl), 4122

14 Martínez-Carmona, M. et al. (2018) Lectin-conjugated pH-responsive mesoporous silica nanoparticles for targeted bone cancer treatment. Acta Biomater. 65, 393-404

15 Vallet-Regí, M. et al. (2018) Mesoporous silica nanoparticles for drug delivery: current insights. Molecules 23, 47

16 Fang, Z. et al. (2019) Hyaluronic acid-modified mesoporous silica-coated superparamagnetic Fe3O4 nanoparticles for targeted drug delivery. Int. J. Nanomed. 14, 5785

17 Luo, W. et al. (2019) Formation of enzymatic/redox-switching nanogates on mesoporous silica nanoparticles for anticancer drug delivery. Mater. Sci. Eng. C 100, $855-861$

18 Zhang, L. et al. (2019) Mesoporous gold nanoparticles for photothermal controlled anticancer drug delivery. Nanomedicine 14, 1443-1454

19 Zhao, P. et al. (2018) TPGS functionalized mesoporous silica nanoparticles for anticancer drug delivery to overcome multidrug resistance. Mater. Sci. Eng. C 84, 108-117

20 Naz, S. et al. (2019) Enzyme-responsive mesoporous silica nanoparticles for tumor cells and mitochondria multistage-targeted drug delivery. Int. J. Nanomed. 14, 2533

21 Lin, J. et al. (2018) PEGylated lipid bilayer coated mesoporous silica nanoparticles for co-delivery of paclitaxel and curcumin: Design, characterization and its cytotoxic effect. Int. J. Pharm. 536, 272-282

22 Dilnawaz, F. (2018) Multifunctional mesoporous silica nanoparticles for cancer therapy and imaging. Curr. Med. Chem. 26, 5745-5763

23 Zhou, Y. et al. (2018) Mesoporous silica nanoparticles for drug and gene delivery. Acta Pharm. Sin. B 8, 165-177

24 Maeda, H. et al. (2013) The EPR effect for macromolecular drug delivery to solid tumors: improvement of tumor uptake, lowering of systemic toxicity, and distinct tumor imaging in vivo. Adv. Drug Deliv. Rev. 65, 71-79

25 Yang, Y. and Yu, C. (2016) Advances in silica based nanoparticles for targeted cancer therapy. Nanomedicine 12, 317-332 
26 Poonia, N. et al. (2017) Mesoporous silica nanoparticles: a smart nanosystem for management of breast cancer. Drug Discov. Today 23, 315-322

27 Watermann, A. and Brieger, J. (2017) Mesoporous silica nanoparticles as drug delivery vehicles in cancer. Nanomaterials 7, 189

28 Meng, H. et al. (2011) Use of size and a copolymer design feature to improve the biodistribution and the enhanced permeability and retention effect of doxorubicinloaded mesoporous silica nanoparticles in a murine xenograft tumor model. ACS Nano 5, 4131-4144

29 Monem, A.S. et al. (2014) Mesoporous silica coated gold nanorods loaded doxorubicin for combined chemo-photothermal therapy. Int. J. Pharm. 470, 1-7

30 Xiao, D. et al. (2014) A dual-responsive mesoporous silica nanoparticle for tumortriggered targeting drug delivery. Small 10, 591-598

$31 \mathrm{Li}$, L. et al. (2012) Polyvalent mesoporous silica nanoparticle-aptamer bioconjugates target breast cancer cells. Adv. Healthc. Mater. 1, 567-572

32 Chen, F. et al. (2018) General synthesis of silica-based yolk/shell hybrid nanomaterials and in vivo tumor vasculature targeting. Nano Res. 11, 4890-4904

33 Fang, W. et al. (2014) pH-controllable drug carrier with SERS activity for targeting cancer cells. Biosens. Bioelectron. 57, 10-15

34 Gary-Bobo, M. et al. (2011) Mannose-functionalized mesoporous silica nanoparticles for efficient two-photon photodynamic therapy of solid tumors. Angew. Chem. Int. Ed. 50, 11425-11429

35 Gayam, S.R. and Wu, S. (2014) Redox responsive pd (II) templated rotaxane nanovalve capped mesoporous silica nanoparticles: a folic acid mediated biocompatible cancer-targeted drug delivery system. J. Mater. Chem. B 2, 7009-7016

36 Feng, Y. et al. (2016) The application of mesoporous silica nanoparticle family in cancer theranostics. Coord. Chem. Rev. 319, 86-109

37 Zhu, C. et al. (2014) Cell microenvironment stimuli-responsive controlled-release delivery systems based on mesoporous silica nanoparticles. J. Food Drug Anal. 22, 18-28

38 (2019) Targeted and stimuli-responsive mesoporous silica nanoparticles for drug delivery and theranostic use. I Biomed Mat Res A 107, 2643-2666

39 et al. (2018) Hollow mesoporous silica@metal-organic framework and its applications for pH-responsive doxorubicin drug delivery. ChemMedChem 13, $400-405$

40 et al. (2018) Recent advances in nanoparticle-based cancer drug and gene delivery. Adv. Cancer Res. 137, 115-170

41 Popova, M. etal. (2018) Polymer-coated mesoporous silica nanoparticles for controlled release of the prodrug sulfasalazine. J. Drug Deliv. Sci. Technol. 44, 415-420

42 Wen, J. et al. (2017) Diverse gatekeepers for mesoporous silica nanoparticle based drug delivery systems. Chem. Soc. Rev. 46, 6024-6045

43 Wong, R.C. et al. (2017) Encapsulating pH-responsive doxorubicin-phthalocyanine conjugates in mesoporous silica nanoparticles for combined photodynamic therapy and controlled chemotherapy. Chemistry 23, 16505-16515

44 Kim, M. et al. (2011) Facile synthesis of monodispersed mesoporous silica nanoparticles with ultralarge pores and their application in gene delivery. ACS Nano $5,3568-3576$

45 Gisbert-Garzarán, M. et al. (2017) pH-responsive mesoporous silica and carbon nanoparticles for drug delivery. Bioengineering 4, 3

46 Du, P. and X, et al. (2015) Layer-by-layer engineering fluorescent polyelectrolyte coated mesoporous silica nanoparticles as $\mathrm{pH}$-sensitive nanocarriers for controlled release. Appl. Surf. Sci. 345, 90-98

47 Xin, Y. et al. (2017) Recent progress on nanoparticle-based drug delivery systems for cancer therapy. Cancer Biol. Med. 14, 228

48 Palanikumar, L. et al. (2015) Noncovalent polymer-gatekeeper in mesoporous silica nanoparticles as a targeted drug delivery platform. Adv. Funct. Mater. 25, 957-965

49 Tian, L. et al. (2019) Hollow mesoporous carbon modified with cRGD peptide nanoplatform for targeted drug delivery and chemo-photothermal therapy of prostatic carcinoma. Colloids Surf. A Physicochem. Eng. Asp. 570, 386-395

50 Singh, S. et al. (2016) Lead phytochemicals for anticancer drug development. Front. Plant Sci. 7, 1667

51 Frederiks, C.N. et al. (2015) Genetic polymorphisms and paclitaxel-or docetaxelinduced toxicities: a systematic review. Cancer Treat. Rev. 41, 935-950

52 Olov, N. et al. (2018) Combinational drug delivery using nanocarriers for breast cancer treatments: a review. J. Biomed. Mater. Res. A 106, 2272-2283
53 Krishna, R. and Mayer, L.D. (2000) Multidrug resistance (MDR) in cancer: mechanisms, reversal using modulators of MDR and the role of MDR modulators in influencing the pharmacokinetics of anticancer drugs. Eur. J. Pharm. Sci. 11, 265-283

54 Kirtane, A.R. et al. (2013) Exploiting nanotechnology to overcome tumor drug resistance: challenges and opportunities. Adv. Drug Deliv. Rev. 65, 1731-1747

55 Sayed, E. et al. (2018) Electrosprayed mesoporous particles for improved aqueous solubility of a poorly water soluble anticancer agent: In vitro and ex vivo evaluation. J. Control. Release 278, 142-155

56 Wong, M.C. et al. (2017) International incidence and mortality trends of liver cancer: a global profile. Sci. Rep. 7, 45846

57 Chi, X. et al. (2019) Targeted arsenite-loaded magnetic multifunctional nanoparticles for treatment of hepatocellular carcinoma. Nanotechnology 30, 175101

58 Wang, J. et al. (2019) Combined chemo/photothermal therapy based on mesoporous silica-au core-shell nanoparticles for hepatocellular carcinoma treatment. Drug Dev. Ind. Pharm. 45, 1487-1495

59 Bhavsar, D. et al. (2019) Formulation and development of smart pH responsive mesoporous silica nanoparticles for breast cancer targeted delivery of anastrozole: In vitro and in vivo characterizations. Microporous Mesoporous Mater. 279, 107-116

60 Perera, M. et al. (2019) Gallium-68 prostate-specific membrane antigen positron emission tomography in advanced prostate cancer-updated diagnostic utility, sensitivity, specificity, and distribution of prostate-specific membrane antigen-avid lesions: a systematic review and meta-analysis. Eur. Urol. 77, 403-417

61 Du, D. , Fu, H.J. , Ren, W.W. , Li, X.L. and Guo, L.H. (2020) PSA targeted dualmodality manganese oxide-mesoporous silica nanoparticles for prostate cancer imaging. Biomed. Pharmacother. 121, 109614

62 Liu, C. et al. (2019) Cancer cell membrane-cloaked mesoporous silica nanoparticles with a pH-sensitive gatekeeper for cancer treatment. Colloids Surf. B Biointerfaces $175,477-486$

63 Rivero-Buceta, E. et al. (2019) PSMA-targeted mesoporous silica nanoparticles for selective intracellular delivery of docetaxel in prostate cancer cells. ACS Omega 4, 1281-1291

64 Taratula, O. et al. (2011) Innovative strategy for treatment of lung cancer: targeted nanotechnology-based inhalation co-delivery of anticancer drugs and siRNA. J. Drug Target. 19, 900-914

65 Li, C. et al. (2017) Combined bortezomib-based chemotherapy and p53 gene therapy using hollow mesoporous silica nanospheres for p53 mutant non-small cell lung cancer treatment. Biomater. Sci. 5, 77-88

66 Tian, B. et al. (2017) pH-responsive poly (acrylic acid)-gated mesoporous silica and its application in oral colon targeted drug delivery for doxorubicin. Colloids Surf. B Biointerfaces 154, 287-296

67 Kumar, B. et al. (2017) Mesoporous silica nanoparticle based enzyme responsive system for colon specific drug delivery through guar gum capping. Colloids Surf. B Biointerfaces 150, 352-361

68 Kesse, S. et al. (2019) Mesoporous silica nanomaterials: versatile nanocarriers for cancer theranostics and drug and gene delivery. Pharmaceutics 11, 77

69 Cui, Y. et al. (2013) Transferrin-conjugated magnetic silica PLGA nanoparticles loaded with doxorubicin and paclitaxel for brain glioma treatment. Biomaterials 34 (33), 8511-8520

$70 \mathrm{Mo}$, J. et al. (2016) Tailoring particle size of mesoporous silica nanosystem to antagonize glioblastoma and overcome blood-brain barrier. ACS Appl. Mater. Interfaces 8, 6811-6825

71 Vallet-Regi, M. et al. (2018) Controlled release with emphasis on ultrasoundinduced release. Enzymes 43, 101-122

72 Mehta, P. et al. (2019) Broad scale and structure fabrication of healthcare materials for drug and emerging therapies via electrohydrodynamic techniques. Adv. Ther. 2 1800024

73 Rasekh, M. et al. (2017) Facile preparation of drug-loaded tristearin encapsulated superparamagnetic iron oxide nanoparticles using coaxial electrospray processing. Mol. Pharm. 14, 2010-2023 